\title{
Beam halo imaging with a digital optical mask
}

\author{
H. D. Zhang, R. B. Fiorito, ${ }^{*}$ A. G. Shkvarunets, and R. A. Kishek \\ Institute for Research in Electronics and Applied Physics, University of Maryland, College Park, Maryland 20742, USA \\ C.P. Welsch \\ University of Liverpool and Cockcroft Institute, Daresbury, United Kingdom
}

(Received 8 March 2012; published 23 July 2012)

\begin{abstract}
Beam halo is an important factor in any high intensity accelerator. It can cause difficulties in the control of the beam, emittance growth, particle loss, and even damage to the accelerator. It is therefore essential to understand the mechanisms of halo formation and its dynamics. Experimental measurement of the halo distribution is a fundamental tool for such studies. In this paper, we present a new high dynamic range, adaptive masking method to image beam halo, which uses a digital micromirror-array device. This method has been thoroughly tested in the laboratory using standard optical techniques, and with an actual beam produced by the University of Maryland Electron Ring (UMER). A high dynamic range (DR $\sim 10^{5}$ ) has been demonstrated with this new method at UMER and recent studies, with more intense beams, indicate that this DR can be exceeded by more than an order of magnitude. The method is flexible, easy to implement, low cost, and can be used at any accelerator or light source. We present the results of our measurements of the performance of the method and illustrative images of beam halos produced under various experimental conditions.
\end{abstract}

DOI: $10.1103 /$ PhysRevSTAB.15.072803

PACS numbers: $29.20 .-c$

\section{INTRODUCTION}

Beam halos are typically observed in particle beams [1,2]. There is no well-accepted or rigorous definition of halo, but it is usually described as a low intensity distribution of particles that are observed at large radii from a more intense centralized portion of the beam, i.e., the "core". Beam halo is associated with emittance growth and thus its presence signifies decreased beam quality. More seriously, halo particles traveling far from the center of the beam can be lost or produce other undesirable effects [3,4]. For example, for a high energy particle beam, lost particles can cause nuclear activation, damage to beam line components, and an increase in the radiation background. For positively charged beams, the secondary electron emission from the impact of halo particles can result in an electron cloud around the beam, which complicates the beam dynamics $[5,6]$. Moreover, since halos are present in the beam phase space, simple apertures that can remove halo particles at a particular beam line location are not effective [7].

A number of theories have been developed and simulation studies have been performed [8-11] to describe the formation of beam halo. These studies have shown that there are many factors which can contribute [12], e.g., intrabeam scattering, collective instabilities, misalignments, magnet

\footnotetext{
*rfiorito@umd.edu
}

Published by the American Physical Society under the terms of the Creative Commons Attribution 3.0 License. Further distribution of this work must maintain attribution to the author(s) and the published article's title, journal citation, and DOI. errors, noise, and resonances associated with both intrinsic incoherent processes and collective space charge forces. However, despite a wealth of theoretical and simulation studies, the mechanisms controlling the formation of halo and their interactions are not well understood. Moreover, few experimental studies of halos have been performed to guide and enlighten theory and simulation [3].

Experimental studies of halos are hampered by the high dynamic range (DR), DR $>10^{5}$, typically required to meaningfully characterize the halo particle distribution. Several approaches have been used to measure halo. These include (1) mechanical devices such as a wire scanners and beam scrappers, which are used to detect halo particles electronically or via induced radiation [13]; (2) indirect or secondary emission monitors, which monitor particles or induced radiation produced when the beam interacts with residual or purposely injected gas [14]; and (3) direct imaging of optical radiation that is produced by the beam interacting with a target or magnetic field and that is linearly proportional to the beam charge distribution, e.g., phosphor, transition, synchrotron, edge, and undulator radiation. In this paper we focus our attention on the direct imaging of the halo distribution produced by radiation from a phosphor screen.

The DR of most imaging techniques is usually limited by the camera sensor (e.g. CCD cameras) to several orders of magnitude. To extend the DR, a number of strategies have been suggested. One of these [15] is to initially use a neutral density (ND) filter to reduce the core intensity so that it does not saturate the camera sensor. Then the filter is removed, allowing the core intensity to saturate the camera 
sensor, in order to view the less intense wings of the distribution. However, this method is problematic because the light from the bright core is scattered in the optics as well as in the sensor; this scattered light can contaminate the measurement of the true halo distribution. There is an additional problem with sensitive sensors such as CCD's and especially intensified CCD's. Blooming in the CCD (charge spill to neighboring pixels) and electron avalanche in the intensifier can disrupt, or at least influence, the measurement and possibly damage the sensor [15]. Advanced charge injection device cameras do not suffer blooming and have the advantage of large intrinsic dynamic range $\left(\mathrm{DR} \geqslant 10^{5}\right)$ [16]. However, these devices require very long acquisition times to achieve a very high dynamic range, cannot deal with very high light intensities and, in any case, light scattering and diffraction of light in the input optics can limit and compromise the measurement of the halo.

One approach that has been successful, applies the concept of solar coronagraphy to image beam halo [17]. The method usually employs transmissive optics and a fixed size blocking mask to filter out the central area of the beam image, highly polished lenses to avoid scattering, and special apertures (Lyot stops) to partially filter out the diffraction effects produced by the input lens and the blocking mask itself. After these steps are taken, the halo is made more visible by increasing the exposure time of the camera. A DR $>10^{6}$ has been achieved with this method.

We have taken the idea of beam coronagraphy a step further by employing a commercially available digital micromirror device (DMD) to generate the core blocking mask. The primary advantages of our technique over conventional beam coronagraphy are: (1) the blocking mask produced on the DMD can be simply programmed to conform (adapt) to an arbitrary shape of the beam core; (2) the intense light from the core can be rejected with very high efficiency, i.e., reflected at a large angle away from the optical path before it reaches the camera optics, which avoids light scattering inherent to the standard transmission coronagraph technique; and (3) the method can, in principle, achieve a high effective dynamic range with a modest dynamic range, low cost CCD camera. The effect of diffraction and other optical effects such as scattering, and aberrations, which contribute to the point spread function (PSF) of the imaging system, are still present and can, in principle, limit the measurable dynamic range. However, as we will see below, the measured PSF of our optical system does not limit the DR of our measurements.

DMD technology has been highly developed by Texas Instruments Inc. (TI) and is used primarily in high definition video displays and projectors. DMD's are also available for research and development purposes in the form of "kits" with a number of different DMD formats and mirror reflectivities optimized for visible, UV and IR bands. Each kit includes a DMD, controller board, a universal serial bus interface and software for computer control of the device. In the experiments described in this paper we use the DMD "Discovery 1100 and 4100" kits manufactured by TI [18]. Each of these includes an extended graphics array format, $14.4 \mathrm{~mm} \times 10.8 \mathrm{~mm} \mathrm{DMD}$ consisting of $1024 \times 768$ pixels $(13.68 \times 13.68$ micron $)$ with micromirror reflectivity optimized for visible wavelengths.

Each pixel of the DMD array can be individually addressed electronically by a complementary metal-oxide semiconductor substructure and rotated $\pm 12^{\circ}$ about the diagonal corresponding to an "on" or "off" state, when a positive or negative voltage is applied to electrodes located beneath the two opposite corners of the micromirror. In the "on" or $+12^{\circ}$ state, incident light is reflected in a direction $24^{\circ}$ with respect to the incident rays, while in the "off" state, the light is directed $48^{\circ}$ away from this path. Thus, by instructing specific micromirrors to "flip" the DMD can be used as a programmable spatial filter.

The filtering ability of DMD and its capability to produce a high effective dynamic range has been demonstrated by using it to measure the intensity profile of a low power He-Ne laser with a standard 8-bit CCD camera [19]. The results showed an effective $\mathrm{DR} \sim 10^{5}$. These laser studies indicated that the DMD masking method could potentially be more useful than a fixed spatial mask, such as used in the coronagraphy technique described above, to image the halo of a charged particle beam with high dynamic range. It was also observed that the DMD produces a crosslike diffraction pattern, characteristic of an array of rectangular apertures, when illuminated by the laser; i.e., the DMD acts like a 2D grating, and the question arose as to how this might effect the image and measurement of beam halo. Our initial experiments, which used the DMD in a real imaging system with nonmonochromatic light, showed that a high DR image of the beam could be achieved with this device and that the diffraction effects were not significant [20].

In this paper, we report the results of more detailed measurements, which verify and add to our previous results. Furthermore, we describe here in detail the optical compensations required to effectively use the device in an imaging system, the design and operation of our optics, measurements of the dynamic range and performance of the imaging system in the laboratory, as well as present the results of our latest measurements of beam halos at the University of Maryland Electron Ring (UMER) [21].

We emphasize that the halos that we have observed at UMER were empirically generated and cannot be presently understood in terms of theoretical models. This will only be possible when systematic studies of halo generation are undertaken. Therefore, the results presented in this work are intended only to illustrate the utility of our technique and to demonstrate the optical performance of the halo imaging system. The results presented here, as well as preliminary results recently obtained at the JLAB 
$100 \mathrm{MeV}$ Energy Recovery Linac and the SLAC/SPEAR3 $3 \mathrm{GeV}$ synchrotron [22,23], indicate that a DR $>10^{5}$ can be achieved with this technique on the beam itself. Moreover, the available DR of the optical systems we have developed at both these facilities has been shown to be $\sim 10^{7}$, so that it may be possible to extend the currently measured DR on the beam by one or two additional orders of magnitude.

\section{EXPERIMENTAL TECHNIQUE}

\section{A. Generic optical imaging system}

Based on the properties of DMD described above, we have designed a flexible optical system that is low cost and easy to employ to image the beam of any accelerator with a high DR. Here the "light source" is any incoherent radiation source which can be used to image the beam. The essential features of the design are schematically shown in Fig. 1.

The setup shown in Fig. 1 can be considered as two optical channels: (1) the source, lens L1 and the DMD surface, which is oriented perpendicular to the optical axis and is the first image plane; and (2) the tilted DMD image plane, considered as a new source, lens L2 and the CCD sensor, which is the second image plane. Note that the DMD in the first channel is perpendicular to the optical axis. This allows us to easily align the entire system. The DMD is mounted on a combination rotation-mirror mount to facilitate the alignment. The first channel is aligned by setting the DMD in a floating state with all the micromirrors nominally flat. An alignment laser can then be retroreflected from the DMD back to the source. When all the micromirrors are turned $+12^{\circ}$, the laser can subsequently be reflected off mirror M1, which facilitates the alignment of the second channel. Two rotational compensations are required to use this system to image the source: (1) the DMD must be rotated about the optical axis by $45^{\circ}$; (2) the camera sensor must be rotated in the horizontal plane by the so-called Scheimpflug angle. The details of these compensations will be described later in this chapter and in the Appendix.

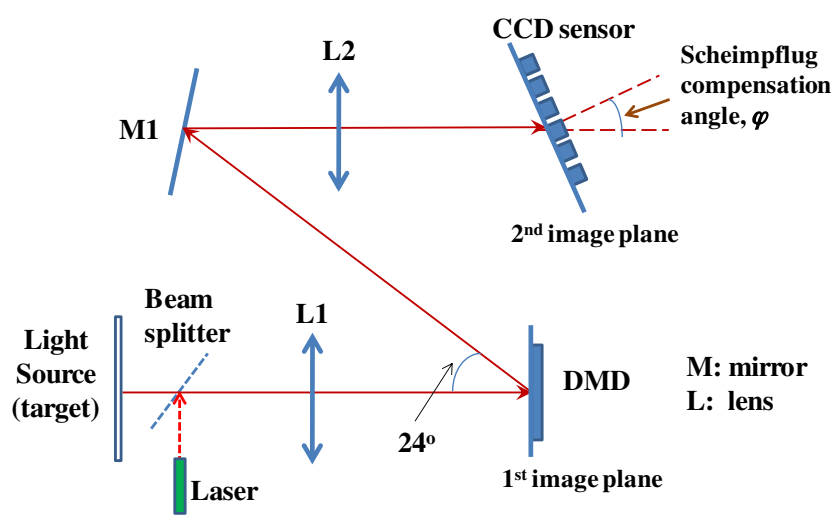

FIG. 1. Schematic of halo imaging optics using DMD.

\section{B. Optics setup at UMER}

The optical system we used for UMER is essentially the same as shown in Fig. 1 except that two lenses are used in each channel in order to separately adjust the magnification in each channel, and additional mirrors are incorporated into the design to meet space constraints. The radiation source at UMER is a $31.75 \mathrm{~mm}$ diameter glass screen coated with P-43 phosphor $\left(\mathrm{Gd}_{2} \mathrm{O}_{2} \mathrm{~S}\right.$ : Tb), which has an emission peaked at $545 \mathrm{~nm}$ and a response time of $1.6 \mu \mathrm{s}$. The screen is oriented at $90^{\circ}$ with respect to the beam direction and the light is directed out of a vacuum cross through an optical port by a front surfaced mirror located a few $\mathrm{cm}$ from the phosphor screen. The location and properties of each optical component in the system are listed in Table I. Here $L$ in the second column is the distance from the phosphor screen.

\section{First optical channel}

One of the design goals for the first optical channel (i.e. the source to the DMD) is to achieve the best image resolution of the phosphor screen on the DMD. To accomplish this we adjust the magnification so that the image of the phosphor screen fills the smallest dimension of the DMD chip, which is a rectangle, $14.3 \mathrm{~mm} \times$ $10.8 \mathrm{~mm}$. Thus, the desired magnification for the first optical channel, $m=10.8 \mathrm{~mm} / 32 \mathrm{~mm}=0.338$. The use of two lenses in the first channel allows us to achieve this value and focus the image onto the DMD within the physical size constraints of our setup. In practice, we chose readily available achromatic lenses, L1 $(D=75 \mathrm{~mm}, f=200 \mathrm{~mm})$ and L2 $(D=50 \mathrm{~mm}$, $f=200 \mathrm{~mm}$ ), and manually adjusted them to achieve focus of the target onto the surface of the DMD. Since there is light scattering from the aluminum edge of the DMD chip holder that may affect image quality at the DMD boundary, we slightly reduced the image size on the surface of DMD to avoid this. Thus, the final magnification is set at $m=0.270$, which is little smaller than the one calculated above.

The rotation axis of each micromirror of the DMD lies along the surface diagonal. Then if the DMD is positioned so that the array's vertical axis is normal to the horizontal plane, the micromirrors on the chip will reflect the incident light out of the horizontal plane. In order to compensate for this, we rotate the DMD by $45^{\circ}$ about the axis perpendicular to the plane of the micromirrors in order to make the rotation axis of each micromirror coincide with the vertical as shown in Fig. 2. As a result, regardless of the orientation of the micromirrors, the light path is maintained in the horizontal plane. This means that the center of all the optical components can be set in the same plane. This greatly simplifies the positioning and the alignment of the optics in both channels and is an essential feature of the optics design. 
TABLE I. Parameters of UMER optical component.

\begin{tabular}{lcccc}
\hline \hline Component & $L(\mathrm{~mm})$ & Focal length $(\mathrm{mm})$ & Diameter $(\mathrm{mm})$ & Description \\
\hline L1 & 600 & 200 & 75 & Achromat \\
L2 & 730 & 200 & 50 & Achromat \\
DMD & 826 & & & \\
M2 & 882 & & 50 & Front surface mirror \\
L3 & 974 & 100 & 50 & Achromat \\
M3 & 1054 & & 50 & Front surface mirror \\
L4 & 1094 & 200 & 50 & Achromat \\
CCD & 1186 & & & PIMAX2 \\
\hline \hline
\end{tabular}

\section{Second channel}

The camera we use is a 16 bit, variable gain, gated, cooled intensified CCD with $512 \times 512$, square pixels (PIMAX2 manufactured by Princeton Instruments Inc.). This type of camera is not essential for the imaging system, but its features are convenient for our optical system performance tests and halo imaging experiments. The transverse size of the input intensifier on the PIMAX2 is $15.8 \mathrm{~mm} \times 15.8 \mathrm{~mm}$. A tapered fiber optic bundle reduces the image on the output side of the intensifier to the size of the CCD which is $12.4 \mathrm{~mm} \times 12.4 \mathrm{~mm}$. Thus, the effective size of each pixel is $30.8 \times 30.8$ microns.

In order to have the best spatial resolution of the image on the CCD, we require that the image of the phosphor screen on the input side of the intensifier be as large as possible, but still allow a clear view of the edges of the DMD. The latter are used to calibrate the number of DMD pixels in terms of CCD pixels which is used in the mask generation algorithm discussed below. To achieve these requirements, we set the magnification, $m=1.033$ using lenses L3 (100 mm) and L4 (200 mm). With this magnification and knowledge of the distance between the DMD and the CCD sensor $(d=364 \mathrm{~mm})$, one can calculate the effective focal length, $f_{\text {eff }}=91 \mathrm{~mm}$, of an idealized single lens which could be used to replace the pair L3, L4 of the second optical channel.

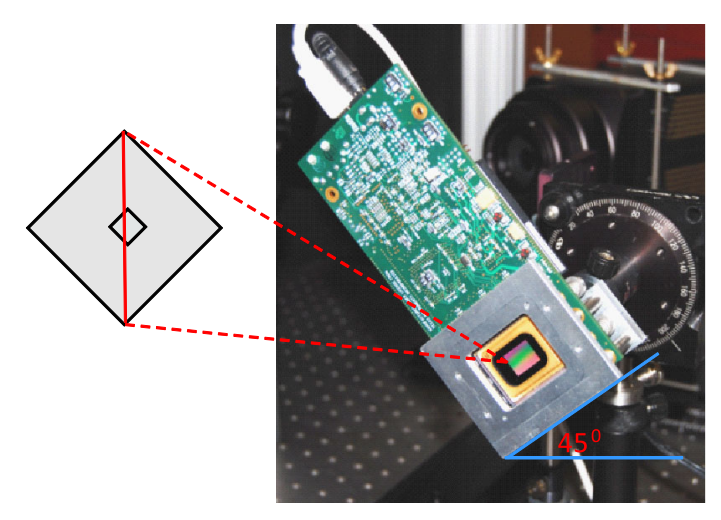

FIG. 2. Picture of rotated DMD (Discovery 1100) and an enlarged sketch of a micromirror.
Note that for the second channel, the image of the secondary source, i.e., the image on the DMD produced by the first channel and viewed by L3 and L4, is not parallel to the effective lens plane but is inclined at $24^{\circ}$. This is due to the fact that the micromirrors, which transmit the desired portion of the beam image from the first channel to the second channel, are flipped $+12^{\circ}$ when activated, so that incoming light rays from the first channel are reflected at twice this angle.

Thus, if we position the camera sensor normal to the optical axis of the second channel, we will obtain an image with a nonuniform focus and magnification in the horizontal plane due to the difference in path lengths of the rays emanating from the DMD. To compensate for this, the camera must be tilted by an angle $\varphi$, the Scheimpflug angle [24], which depends on the tilt angle of the image plane $\left(24^{\circ}\right)$ and the magnification of the first channel.

A full description of the Scheimpflug compensation, including a calculation of the Scheimpflug angle, the depth of field, and the distortion introduced by the compensation are presented in Appendix A. We show there that $\varphi=24.7^{\circ}$ for our system. Empirically judging the best focus and minimal distortion by viewing a gridded test target, we set the angle of our camera to $24^{\circ}$. The difference between these two angles produces a depth of field $a=0.06 \mathrm{~mm}$. However, this is very small compared with the dimension of the DMD and hence is unimportant. In addition, an unavoidable distortion occurs when Scheimpflug compensation is applied. However, since the magnification of the second optical channel $(m=1.033)$ is close to unity, the distortion is also negligible.

\section{Mask generation algorithm}

In order to generate a mask for the beam core, a coordinate transformation and rescaling is necessary, because of the $45^{\circ}$ orientation of DMD and the different number of pixels in the DMD and the CCD sensor. The algorithm is shown schematically in Fig. 3.

The procedure is as follows. First we take a calibration picture to view the edges of the DMD chip on the CCD, and thus determine the DMD chip size $(\Delta x, \Delta y)$ and the transformation equations describing the edges of DMD chip in 


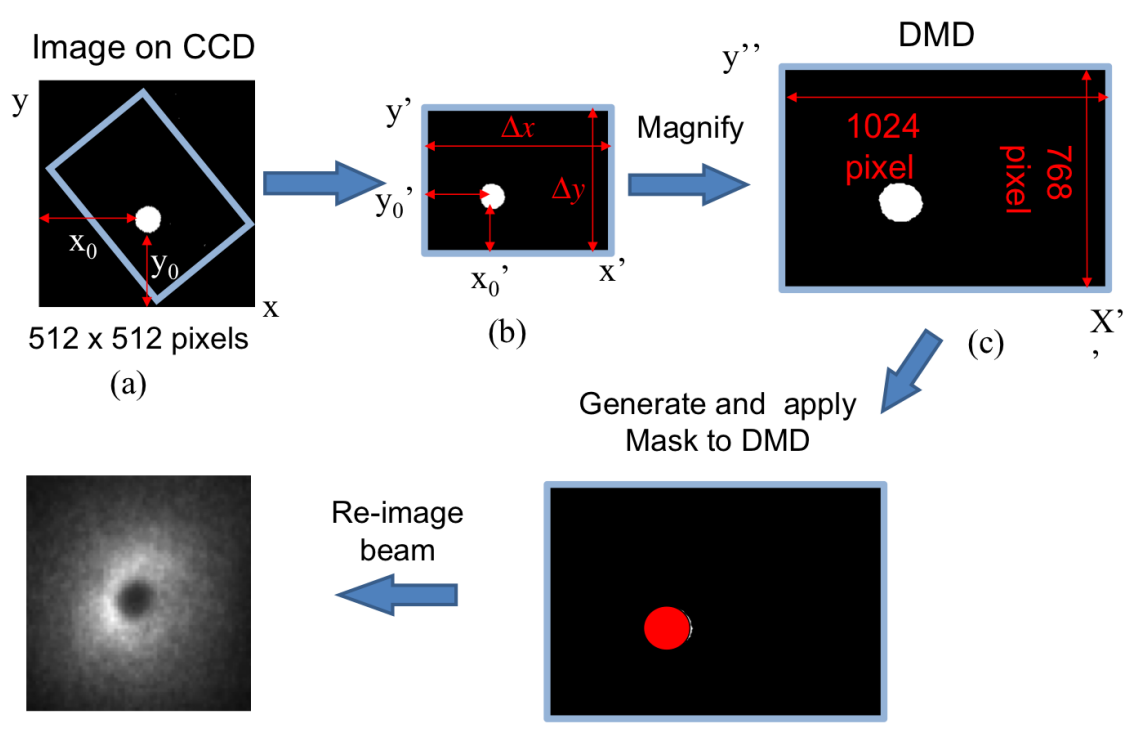

(e)

(d)

FIG. 3. Schematic of mask generation algorithm.

terms of the CCD coordinates $(x, y)$ as shown in Fig. 3(a). Then, we generate a single bit transitional picture, $\Delta x \times \Delta y$ in size, with all pixels "on" set to 0 and all pixels "off" set to 1 . After we take a beam picture, any point of interest $\left(x_{0}, y_{0}\right)$ can then be transformed to a transitional picture with coordinates $\left(x_{0}^{\prime}, y_{0}^{\prime}\right)$; all points of the transitional image can be written in terms of coordinates $\left(x^{\prime}, y^{\prime}\right)$, by calculating the distance between the point and the edges of the DMD. We then choose selected discrete points on the beam image in this manner based on the intensity or position in the primary image that we wish to reject and set that pixel to the "off" or $-12^{\circ}$ state to generate a mask which will reject, i.e., block the beam core as is shown as the red area in Fig. 3(d). There are several ways to define the mask. For example, we can select points in the core, by (1) specifying a particular geometric area (e.g. a circular disk) visually to define the core, or (2) by prescribing a chosen intensity threshold value to define the core.

Note that the area $(\Delta x \times \Delta y)$ is specified in units of the pixels of the CCD, which is much smaller than the size of DMD. Thus, in the next step, shown in Fig. 3(c), we linearly magnify the transitional picture by the ratio $\Delta x \times \Delta y$ divided by the area of the DMD chip, and then generate the final 1-bit mask.

Once the mask is applied to the DMD, the masked beam is reimaged on the CCD camera and a final image is generated by integrating by the number of beam pulses required to bring the peak intensity of the final image close to the saturation level of the camera sensor. The gate feature of the PIMAX2 is utilized to do this by setting the gate width to slightly exceed the UMER beam pulse width (100 ns) and accumulating images for a set number of gates, which correspond to the required number of beam pulses. This feature minimizes the background from stray light sources.

\section{EXPERIMENT RESULTS}

\section{A. Optics bench tests}

\section{DMD diffraction effects}

As noted above, the DMD behaves like a 2D optical grating. If illuminated by a single wavelength laser source, a crosslike diffraction pattern similar to that of a rectangular mesh will be observed. In addition, when all the micromirrors are flipped by $+12^{\circ}$ the DMD becomes a blazed grating with the central order reflected in the direction $+24^{\circ}$ in the horizontal plane with respect to the incident laser beam. When the DMD is rotated by $45^{\circ}$, the diffraction pattern also correspondingly rotates as is shown in Fig. 4(a). To obtain this picture the central order was suppressed to prevent saturation of the CCD imager. When a uniform source of white light illuminates this "blazed" grating, the light is further dispersed in the horizontal plane producing the Fraunhofer pattern shown in Fig. 4(b). Both pictures were obtained by imaging the light diffracted by

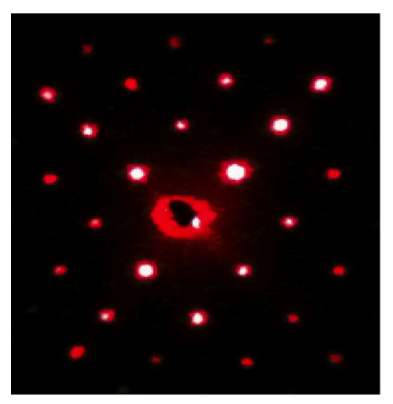

(a)

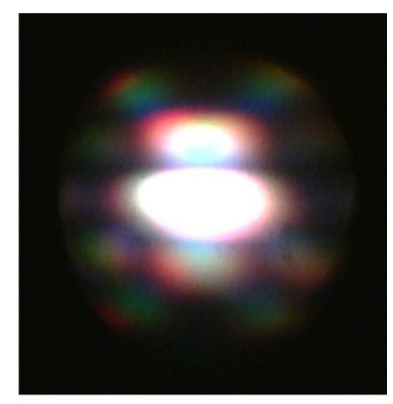

(b)
FIG. 4. (a) Single wavelength diffraction pattern; and (b) white light diffraction pattern, both formed from a $45^{\circ}$ rotated DMD with all pixels set at $+12^{\circ}$. 
the DMD (all pixels "on") in the focal plane of a $200 \mathrm{~mm}$ focal length lens.

Note that for the white light diffraction pattern all the orders are smeared in the horizontal plane. This is due to the effect of both wavelength dispersion and overlapping of the light from the central spot and nearby first order diffraction spots. The latter effect is particularly evident in the central and first orders. The picture further makes it clear that most of the light reflected by the DMD is contained in these two orders.

If the incident light on any grating is nonuniform but has a known distribution, i.e., is an "object," any order of the light diffracted by the grating can be used to image the object. Any aperture in the optics will filter out higher order diffraction spots which ultimately will reduce the imaging resolution. So this must be checked for a given application. However, if the numerical aperture of the second channel optics is sufficient to accept the central two orders, most of the light diffracted by the DMD will be relayed into the second channel. This is indeed the case for our optics. We have traced the rays corresponding to the angles of the central and first order diffraction spots with an optics code to ensure that they are not vignetted as they pass through the optics of channel two.

\section{Spatial resolution}

There are several standard techniques that can be used to measure the spatial resolution of an optical system. We measured the resolution of our optical system by imaging a "knife edge" target, which was constructed from a thin, rectangular piece of black anodized aluminum foil (Cinefoil) mounted on a white card, onto the DMD. The card was backlit with an adjustable intensity "white light" source (i.e. an incandescent lamp). In these bench tests we made use of a higher resolution (PIMAX1) camera which has the same size intensifier as the PIMAX2 $(15.8 \times 15.8 \mathrm{~mm})$ but is reduced by a fiber optic bundle to a larger $(1024 \times 1024$ pixels $)$ CCD array. Each pixel of this camera has an effective size of $15.4 \times 15.4$ microns.

To ensure that the DMD plane was in good focus on the camera to begin with, we programmed the DMD to accept a well-known test image, i.e., a black and white checkerboard, which is included in the software supplied by the manufacturer to control the DMD. This pattern is ideal to adjust the focus of the second channel and to test for distortion, since it originates with a few microns of the surface plane of the DMD chip and has multiple sharp black-white boundaries, i.e., the checkers. Once this source was focused onto the camera, we turned all the pixels to the "on" or $+12^{\circ}$ state to reflect the resolution target image from the first channel into the camera light path. We then adjusted the focus of the first channel lens system to produce the sharpest image of the resolution target on the camera, without moving the lenses in the second channel.
Figure 5 shows two views of the resolution target; the left-hand side (a) is a full view of the entire target; the right-hand side (b) is a magnified view of the corner of the black, rectangular portion of the target. Vertical and horizontal line scans of the corner of the black corner of the target at the pixel level show an "S" shaped dispersion curve. Figure 6 shows a horizontal and vertical line scans of the target which are averaged over 4 vertical pixels [see the white box shown in Fig. 5(b)]. To analyze the resolution of the image we assume that the point spread function (PSF) of the optical system can be represented by a 2D Gaussian and convolved this function with the source intensity distribution $A(x, y)$. A line scan across the image, e.g. $I(x)$, the brightness along the linear scan ( $X$ direction) normal to the sharp linear boundary ( $Y$ direction, at $Y=0$ ) is then described by the convolution integral:

$I(x) \propto \iint A\left(x^{\prime}, y^{\prime}\right) \exp \left(-\frac{\left(x-x^{\prime}\right)^{2}}{2 \sigma_{x}^{2}}-\frac{y^{\prime 2}}{2 \sigma_{y}^{2}}\right) d x^{\prime} d y^{\prime}$,

where we assume $A(x, y)=$ const at $X>0$ and $A(x, y)=0$ at $X<0$. A similar expression can be derived for a $Y$ scan. One can easily show that the resulting intensity scans are error functions. Simple fits of the experimental horizontal and vertical scan data to the corresponding error functions and the respective Gaussian functions, which are the derivatives of these functions, are shown in Fig. 6. The half widths of the horizontal and vertical Gaussians are 3.0 and 2.1 , respectively. These widths provide an estimate of the resolution of the entire optical system.

We compared these measured values of the resolution of the DMD optical system to that of a "standard" optical system in which the DMD is replaced by a simple mirror. The major difference in the optics, when the mirror is used, is that Scheimpflug compensation is not required. So for the resolution test with the mirror we rotated the camera back to its initial orientation, i.e., perpendicular to the optical axis of the second optical channel. We then observed the knife edge resolution target and followed the same procedure described above to measure the resolution. The measured half width of the black-white transition

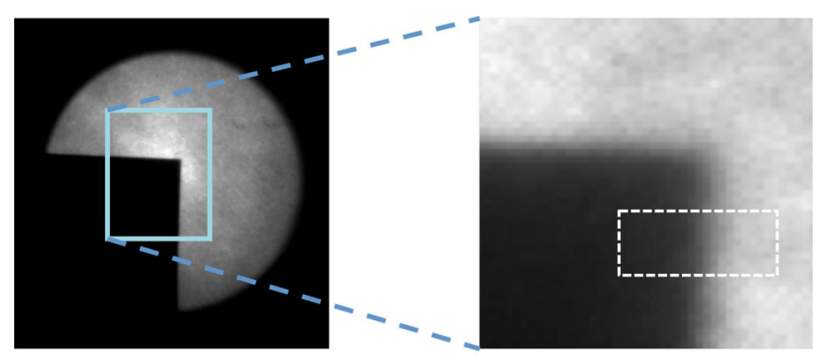

(a)

(b)

FIG. 5. (a) Resolution target; left: full view, right: (b) Expanded $(\times 16)$ view. 

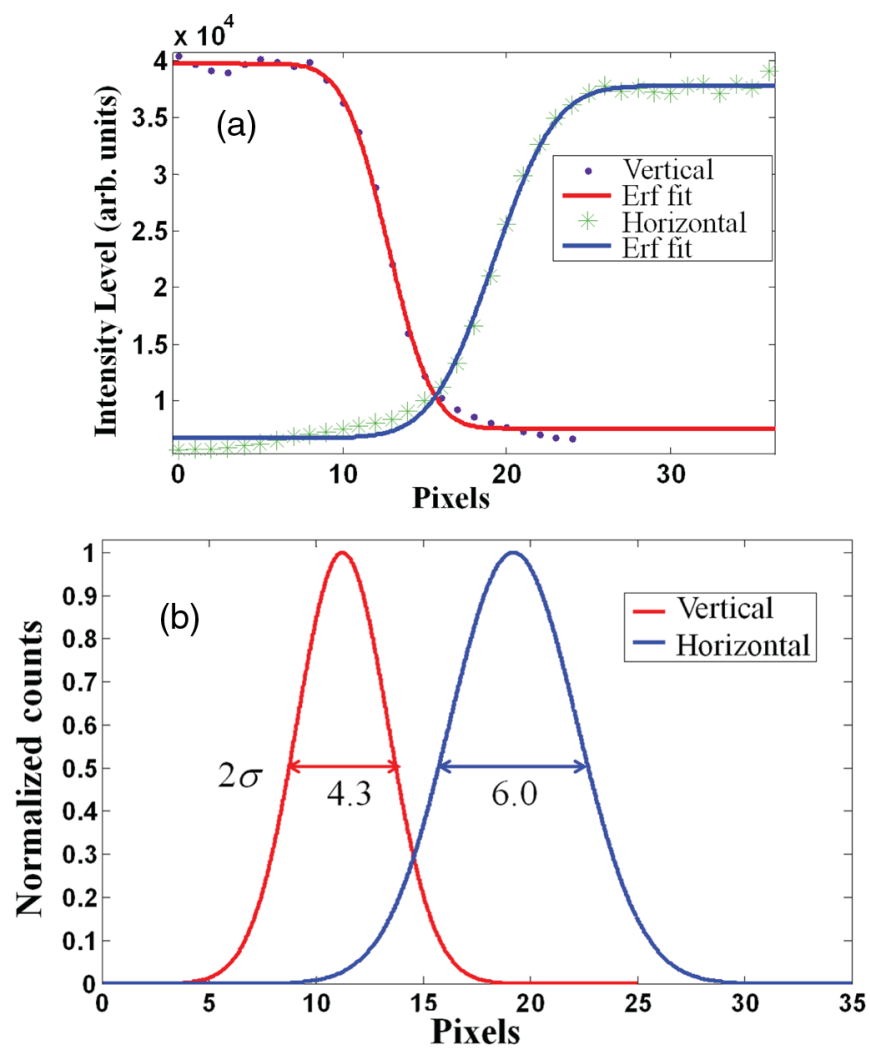

FIG. 6. (top) Horizontal and vertical scan of resolution target corner shown above in Fig. 5; and (bottom) related Gaussian functions.

region in both the horizontal and the vertical directions is $\sigma \sim 3$ pixels, which is approximately the same value measured for the DMD optical system. This indicates that the optical resolution of the DMD optics is essentially the same as that of the standard mirror system.

\section{Single pixel response}

We have also measured the single pixel response of the second optical channel. To accomplish this, a uniform white light source beam is directed onto the entire DMD but with only a single micromirror activated. The light from this single illuminated pixel is imaged onto the CCD via lenses L3 and L4. Figure 7 shows the resulting image (shown as a negative) as well as horizontal and vertical line scans across the image. The scans show that the half width of a Gaussian fit to distribution of intensity in both the horizontal and vertical directions is $\sigma \sim 3$ pixels, which, interestingly, is the same as the measured optical resolution of the entire optical system. This result means that the DMD does not significantly influence the resolution or SF of the optics system. Furthermore, since the resolution of the second channel is comparable to that of the overall system, the width of the PSF of the first channel must be less than that of the second channel or very close to it.

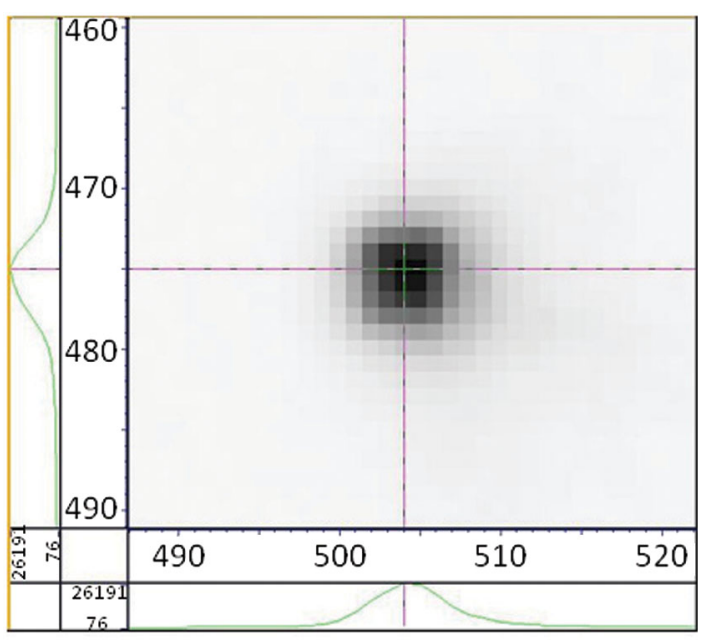

FIG. 7. Response of a single DMD pixel to uniform white light illumination.

\section{High dynamic range measurement of the PSF}

As far as dynamic range (DR) of the imaging system is concerned, it is only necessary to measure the PSF of the first channel. The reason for this is that the second optical channel merely reimages the first image from the DMD plane with or without a mask in place, with an inherently low dynamic range imaging system.

The optical masking technique we will describe below uses a series of images each of which are taken using a ICCD camera with a $\left(\mathrm{DR} \sim 10^{4}\right)$ corresponding to about 16 bits. Thus, the wings of the PSF of the second channel below $10^{-4}$ of the peak intensity of any masked or unmasked image of the beam are not visible to the CCD camera. In fact any contamination of the true beam halo due to the wings of the real PSF will be visible in the first channel and affect image on the DMD. This means that if the wings of the PSF exceed the beam halo in first image they will also exceed the beam halo in the distribution, which is reconstructed using the DMD masking method. Similarly, if the wings of PSF are below the true beam halo level, then this will be the case in the reconstructed image as well. Thus, for all intents and purposes it is sufficient to measure the PSF with high dynamic range for the first channel only.

In order to do this, we used a wideband (white), "pointlike" source with a homogeneous angular distribution that closely mimics that of the phosphor screen used at UMER. A schematic of the measurement system is shown in Fig. 8. The source is a white thread illuminated by light produced by a light-emitting diode (LED) that is $4 \mathrm{~mm}$ in diameter. The LED light is focused (magnification $=0.11$ ) onto the thread by a Nikon camera lens with focal length $f=$ $28 \mathrm{~mm}$ that is oriented so that the normal input aperture of the lens faces the LED. The thread is a very good diffuser and scatters the focused LED light uniformly into a wide angle. We verified this by imaging the angular 


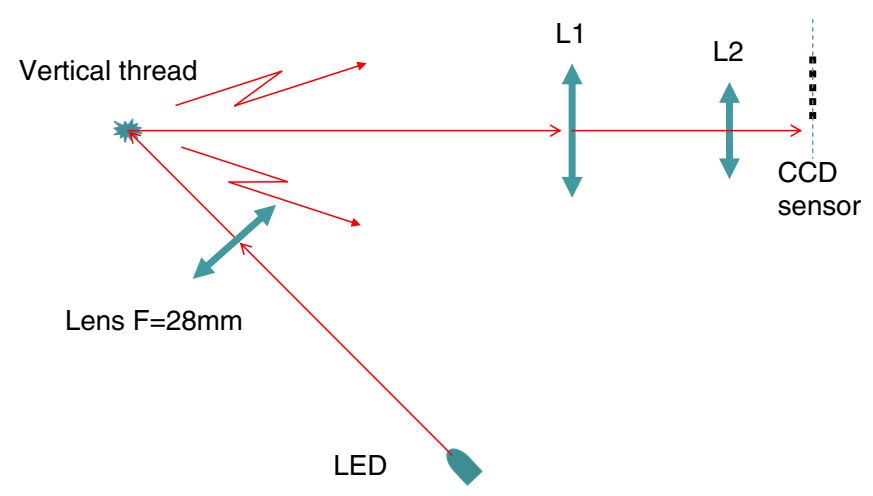

FIG. 8. Sketch of the experimental setup for PSF measurement of the first optics channel.

distribution of the reflected light with a CCD camera placed in the Fourier plane of the first optical channel, which showed a uniform irradiance across the whole acceptance solid angle. The image of the LED on the thread (geometrical size of the source) is $0.45 \mathrm{~mm}$ in length and $0.25 \mathrm{~mm}$ in width corresponding to the diameter of the thread. When the light scattered by the thread is focused onto the CCD sensor by the first optical channel (i.e. lenses $\mathrm{L} 1$ and L2 with magnification $=0.27$ ) the geometrical size of the spot on the intensifier of the PIMAX1 camera that we use for this measurement is $70 \times 120 \mu \mathrm{m}$, or about $5 \times 8$ pixels (each pixel has effective size $15.4 \times 15.4 \mu \mathrm{m}$ ).

We measured the PSF of the first channel by progressively shifting the bright central spot in the image of our source away from the active area of sensor of CCD camera by means of linear actuator and successively applying neutral density filters to attenuate the light to avoid saturating the $\mathrm{CCD}$. With this technique we were able to utilize the whole size of the CCD sensor and achieve a dynamic range DR $\sim 10^{7}$. The results are shown in Fig. 9.

We note that the measured PSF has a much wider FWHM than the PSF, calculated from diffraction theory, i.e., the Airy disk. Furthermore, the intensity of the wings

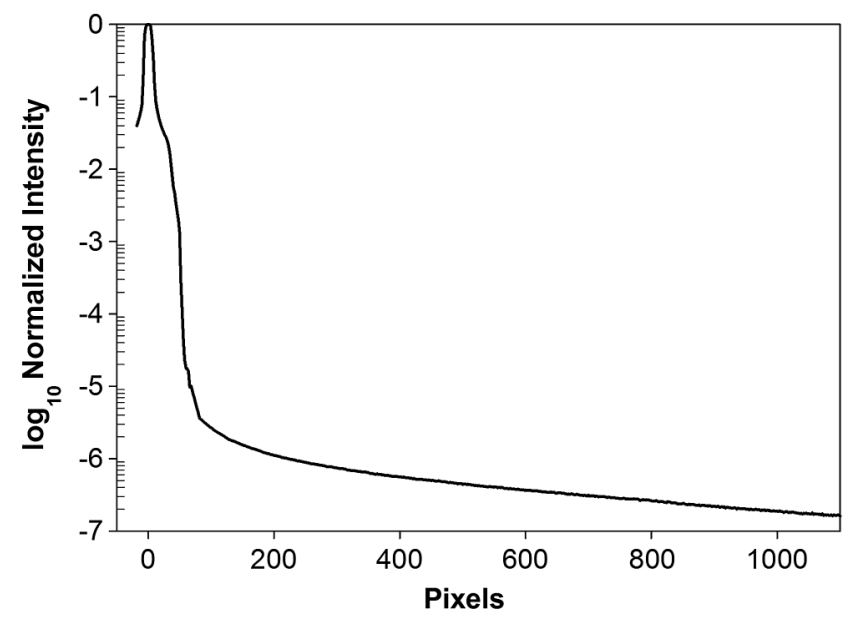

FIG. 9. Measured PSF of the first optical channel. of the measured PSF in the interval $100-500$ pixels is several orders of magnitude greater than the level calculated from diffraction theory. This is due to the combined effects of scattering, aberration, nonuniformities, and diffraction in all the elements in the optical transport. The importance of this measurement will become apparent later when we discuss the dynamic range measurements done using the UMER electron beam.

Also, because of the finite size of our source, the measured PSF shown in Fig. 9 differs from that of a "true point source," especially in the region close to the source. For instance, PSF in Fig. 9 has FWHM $=12$ pixels which is wider than knife edge and "single pixel" measured PSF with FWHM $\sim 5$ pixels. However, at large distances from the source the intensity distribution will be close to that of the "true PSF" because it is not affected by the size of the source at sufficiently large distances, i.e., when the ratio of distance over the source size is large.

\section{B. Beam imaging studies at UMER \\ 1. Characteristics of UMER}

In order to test the DMD imaging method described above with a real beam, we use the University of Maryland Electron Ring (UMER), which is a small compact electron storage ring with a low energy $(10 \mathrm{keV})$ but relatively high beam current $(1-100 \mathrm{~mA})$. It is designed to study the physics of electron beams, in both the emittance and space charge dominated regimes, which can be scaled to lepton beams with higher energies or hadron beams with low beta [21]. UMER is well suited for the experimental study of halos, since (a) it can easily generate beams with and without halos; (b) it has a number of phosphor imaging screens which can generate a substantial amount of light; and (c) the beam is highly stable and thus reproducible over many pulses. This allows us to acquire images via frame integration with minimum error due to shot-to shot variations. Figure 10 shows a schematic layout of UMER.

The electron gun [25] of UMER has a circular thermionic cathode source with a radius of $4 \mathrm{~mm}$. The anode-tocathode distance is $25 \mathrm{~mm}$. The grid is positioned between them, $0.15 \mathrm{~mm}$ away from the cathode. The grid is biased with a negative voltage (bias voltage) with respect to the cathode, to avoid the flow of electrons into the injector, until a positive over-riding beam pulse is applied. During the high level of the beam pulse, the beam current is allowed to flow into the injector of the ring.

Tests of the performance of the optical system with a real beam are done on UMER at diagnostic chamber IC1 (the first injection chamber), using a phosphor screen shown as "S1" in Fig. 10, where the beam core is approximately round. This is achieved by correctly biasing the grid of the UMER triode-type electron gun. In normal operation we set the bias voltage to $-30 \mathrm{~V}$; the beam current pulse has a flattop shape with little fluctuation and the beam is round with almost no halo. However, when we alter 


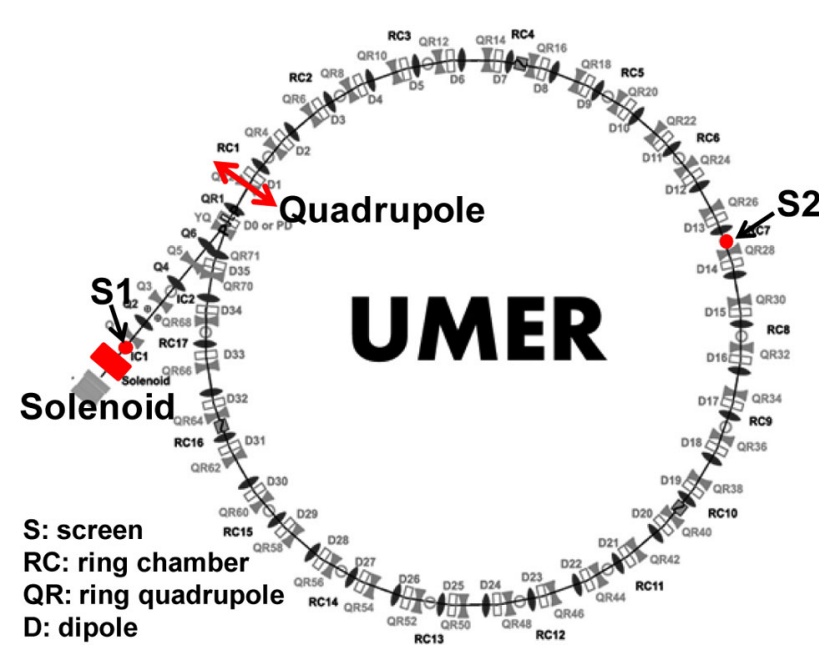

FIG. 10. Schematic layout of UMER.

the grid bias voltage to $-50 \mathrm{~V}$, we observe that the beam current fluctuates with a large variation, which indicates that each longitudinal slice of the whole beam has a different transverse size. When all the slices are integrated over the time of the pulse, a halo is observed. We use this resulting beam to: (1) test the filtering effectiveness of the DMD; (2) test the imaging quality of the optics; and (3) measure the dynamic range of the entire optical system, which is accomplished by changing the solenoid strength to focus the beam as small as possible onto the phosphor screen S1.

\section{Extinction properties of DMD}

We tested the effectiveness of the DMD to reject light away from the desired optical path (i.e. channel 2) in the following way. We first set all the DMD pixels to the "on" state, so the whole image of the beam is transported to the CCD sensor. This image is shown in Fig. 11(a). The peak intensity is about 61500 counts which is close to the saturation level (65 355) of the CCD pixels. The camera was set to integrate 180 beam pulses to reach this level. Next, we set all the pixels of DMD to "off" state. In order to compare with the previous one, we also integrated 180 beam pulses resulting in the image shown in Fig. 11(b). Comparing these two images in the beam region, the image with the all DMD pixels "off" has an intensity level at least $10^{3}$ less than the same region with the pixels "on". Figure 11(b) shows that in the beam region, the intensity level is $\lesssim 50$ counts, i.e., it is close to the noise level. Notice that there are two visible lines with a small number of counts $(\sim 250)$ seen in the image shown in Fig. 11(b). These lines are due to stray light scattered from the edges of the DMD.

\section{Image quality tests}

Second, we did an experiment to test if the DMD affected the beam image quality. Beam images taken with the DMD and with a simple flat mirror were obtained; the results are shown in Fig. 12. Figure 12(a) shows a beam image obtained with the DMD and normalized by the peak intensity; here all of the micromirrors were set to the "on" or $+12^{\circ}$ state and Scheimpflug compensation of the camera was required. Figure 12(b) shows a similarly normalized image obtained using a standard front surface aluminized mirror and no compensation was employed.

Note that the optical elements must be slightly adjusted for each experiment. This causes slight differences in the magnification and number of peak counts for each case as well as a shift of the image on the CCD. However, to the eye the images are nearly indistinguishable. To further compare them, we shifted the image centroids so that they overlapped. Figure 13 shows the horizontal and vertical line scans of the two normalized, shifted images. The line scans are nearly identical and have the same FWHM's. Subtraction of the two normalized, shifted images again shows very small differences.

From this data, coupled with the resolution data described above, which also shows no essential difference
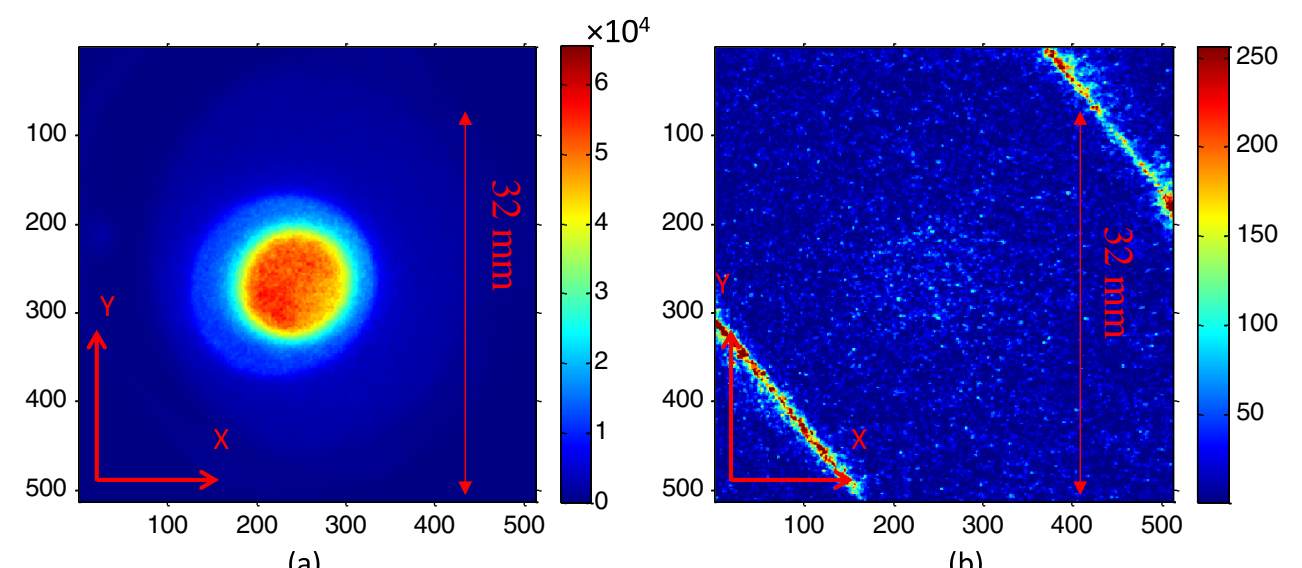

FIG. 11. (a) Image with all DMD pixels set to $+12^{\circ}$; (b) image with all pixels set to $-12^{\circ}$; vertical and horizontal coordinates are given in terms of CCD pixels 


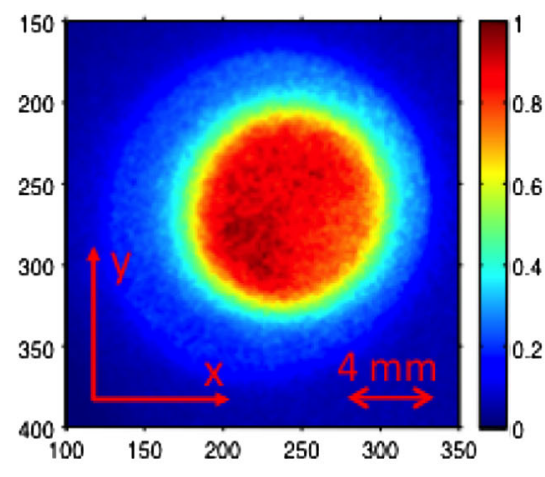

(a)

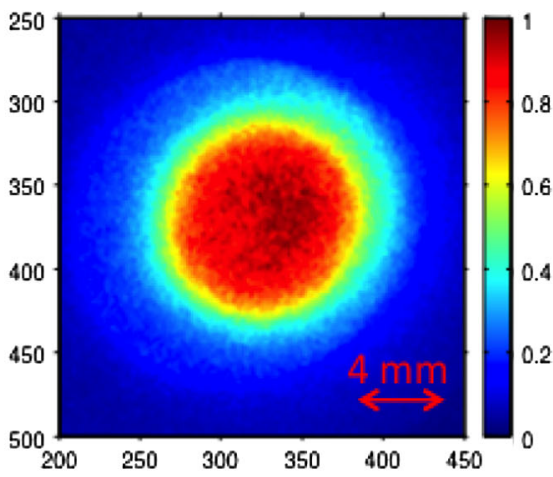

(b)

FIG. 12. Comparison of normalized beam images with (a) all DMD pixels set to "on" and Scheimpflug compensation; (b) simple mirror and no compensation.
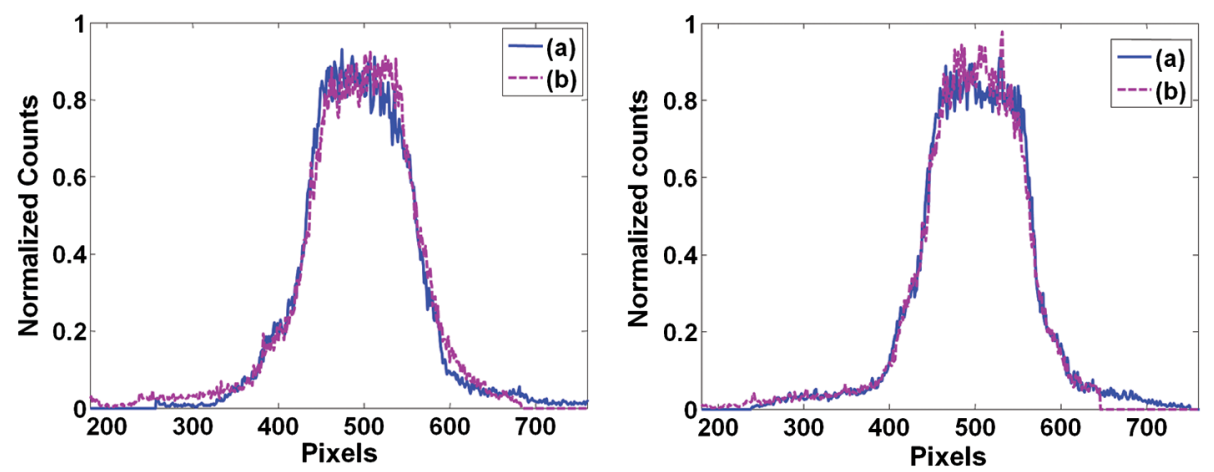

FIG. 13. Left: Horizontal and right: vertical line scans of the two cases shown in Fig. 12.

between the resolutions measured with the DMD and the mirror, we conclude that diffraction and scattering by the DMD mirrors has little if any effect on the quality of the beam image.

\section{Dynamic range measurement with beam}

In order to determine the dynamic range of the entire UMER optical system, we observed an intense beam ( $I=21 \mathrm{~mA}$ ) focused to a $2.85 \mathrm{~mm}$ diameter spot on the phosphor screen, which is the highest current and smallest size that can be achieved with our solenoidal focusing magnet. We then generated a number of circular masks with different radii but a common origin, i.e., the position of the peak intensity of the beam observed without a mask. For each mask, we integrated over the appropriate number of frames (the number written underneath each photo of Fig. 14) that was necessary to bring the peak intensity in the image close to the saturation level of the camera. Note the small highlights visible in the upper left-hand part of pictures number 3 and 4 . These are due to scattering of the phosphor light from the metal edge of the screen. To block out these undesirable highlights we created additional small masks on the DMD, which are seen as black dots on the images number 5 and 6 of Fig. 14.
To obtain a background image, we turned the beam off and integrated for the same number of frames that was used to obtain the beam image. Background subtracted images are shown in Fig. 14. By taking the horizontal line's scans of all beam profiles (note for reference the horizontal red line in Fig. 14) and normalizing by the number of frames of each image, we can present the results as a series of normalized plots as shown in Fig. 15.

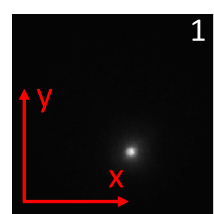

20

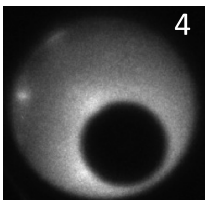

2000

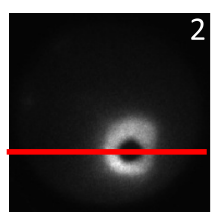

275

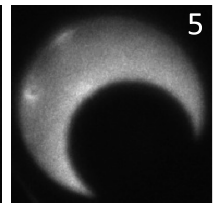

3000

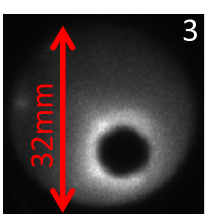
1000

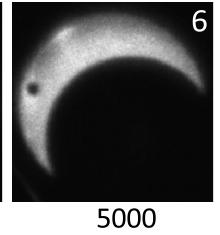

FIG. 14. Images of the focused beam with the DMD set to concentric circular mask of successively larger radii; number below image denotes the number of frame integrations on the ICCD camera. 


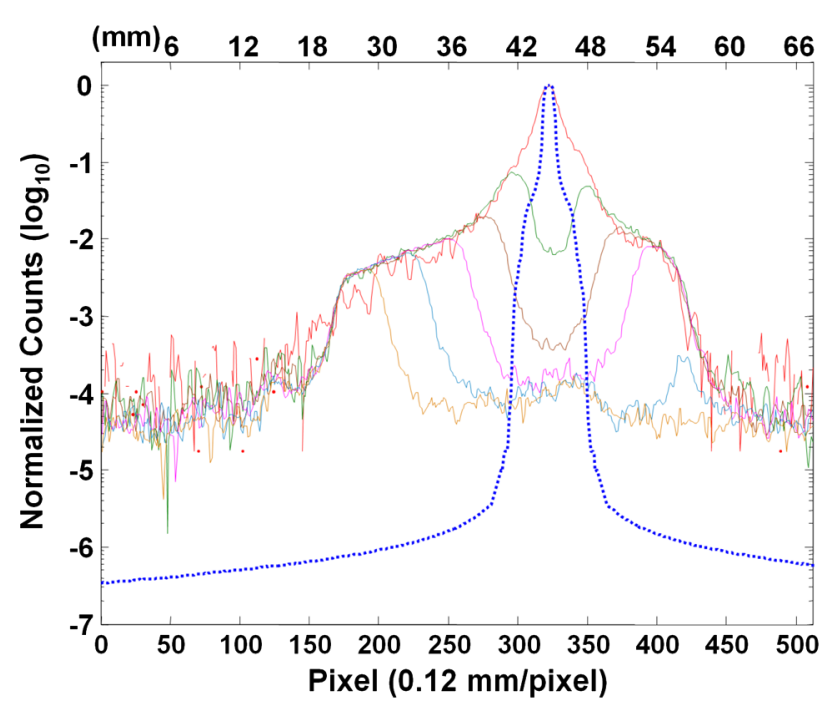

FIG. 15. Normalized horizontal scans of selected beam images presented in Fig. 14; with the measured PSF shown in Fig. 9 (blue dotted line); upper axis: scale at the source plane; lower axis: scale at intensifier plane.

Note the intensity fluctuations in tails of the beam profiles in Fig. 15 decrease as more integration is applied. Moreover, the noise level outside the screen decreases to $\sim 10^{-5}$. Comparison of the recorded electron beam distribution with the measured PSF of the first optical channel (the blue dotted line) makes it clear that the PSF "wings" due to the central, brightest portion of the beam (i.e. the core) do not obscure (overilluminate) the halo region of the beam. Thus for our experimental conditions it was not necessary to use special Lyot stops or highly polished lenses to improve the PSF, as is done in conventional beam coronagraphy, in order to achieve a system DR $\sim 10^{5}$.

Using the data presented in Fig. 14 and normalizing by the number of frames we can reconstruct a high dynamic range beam image as shown in Fig. 16. For this reconstruction, each point in the figure is chosen from an intensity interval, corresponding to the peak value to $1 / 60$ of the peak value, for each image presented in Fig. 14. Then the value of the intensity for each pixel is normalized and converted into standard bitmap format using the relation

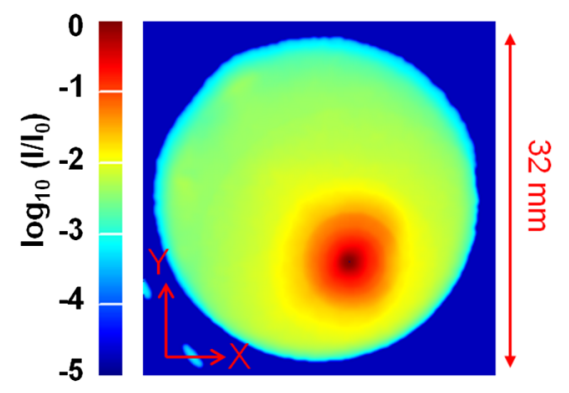

FIG. 16. Reconstructed image showing colorized logarithmic intensity contours of the beam, phosphor screen periphery, and background.
$51 *\left[5+\log _{10}\left(I / I_{0}\right)\right]$, where $I_{0}$ is the absolute maximum value of all the intensities.

Note that we have used only a small part of the DR of each picture in the reconstruction. The means that a camera with a much smaller available DR (e.g. an 8-bit CCD) could be used to achieve the same reconstructed high DR beam image. This was in fact done in earlier laser experiments using the DMD [19]. This is an important feature of our halo imaging technique. Use of a low cost imager immensely reduces the requirements and cost of the optical system needed to do halo measurements.

We note also that our measured DR at UMER is inherently limited by the minimum size of the beam that can be obtained with our solenoid magnet, the maximum current density of the UMER beam, the saturation level of the phosphor, and the size of the screen, i.e. not by the optics or the inherent properties of the DMD itself. Also note that the beam is off centered; this is due to the misalignment of the solenoid with respect to the beam direction.

\section{Beam halo measurements at RC7}

At RC7 (the 7th ring chamber), denoted by screen $\mathrm{S} 2$ in Fig. 10, where the beam distribution is typically not round, we performed experiments to test the adaptability of the masking method to changes in the shape of the beam. In the UMER ring, the beam is not perfectly matched, so the time integrated beam distributions we observe are complex. By varying the quadrupole upstream, for example QR2 (the second ring quadrupole), we can empirically perturb the beam and thereby change the eccentricity of the beam core as well as the halo structure. Under these conditions we generated masks on the DMD that adapt to variations in the core of the electron beam. The results for two different beams with currents of 21 and $6 \mathrm{~mA}$ are shown in Figs. 17 and 18 , respectively.

Figure 17 shows a group of beam images taken with the $21 \mathrm{~mA}$ beam, where the numbers in the lower left are the number of frames integrated and the numbers between rows (a) and (b) are the threshold intensity levels used to generate the mask. We decreased the original quadrupole current strength $\left(I_{Q}\right)$ by $12.4 \%$ and $28.8 \%$ to affect changes in both the beam core and the halo. A decrease in the quadrupole current results in a linear drop in the position of the beam. Row (a) in Fig. 17 shows photos of the beams for three current settings, which indicate that the core gradually enlarges in the $x$ direction and shrinks in the $y$ direction and thus becomes elliptical. Row (b) shows the same beam after applying threshold masks which conform to the variations in the core shapes shown in row (a). We also see that the degreasing quadrupole current strength and thus an increase in the focal length of the quadrupole also affects the halo distribution. Note that more particles are driven out of the core, as shown in Fig. 17, as the size of the high intensity portions of the halo regions become larger. 
(a)
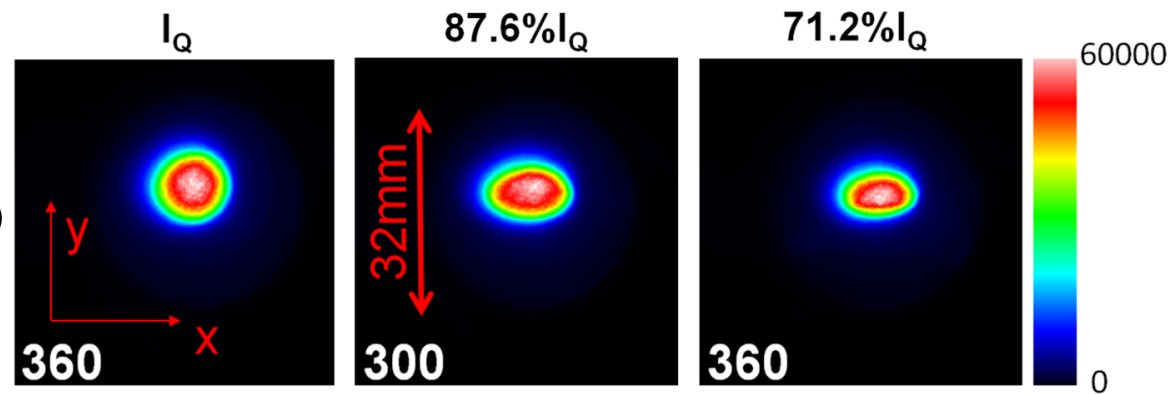

12000

10000

12000

(b)
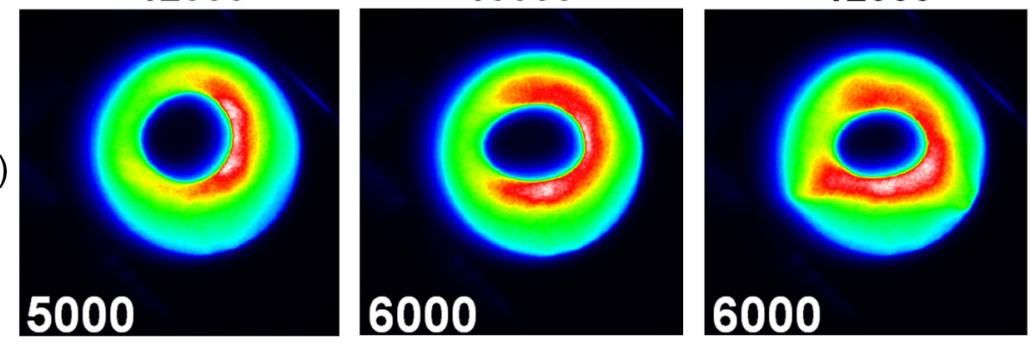

FIG. 17. Comparison of core and halo with changing quadrupole strength. (a) Unmasked beam picture; (b) masked beam picture.

In order to see the full extent of the halo, a much smaller beam with $6 \mathrm{~mA}$ current (radius $=0.875 \mathrm{~mm}$ ) was used. We again decreased the original quadrupole current strength $\left(I_{Q}\right)$ by $17.1 \%, 33.7 \%$, and $50.3 \%$ to see the effect on the beam core and halo. Figure 18 shows the results. Comparing the pictures in row (a), we see the beam centroid gradually moves toward the negative $y$ direction. This may be the result of quadrupole misalignment with respect to the center of beam pipe or beam misalignment. Again as the quadrupole current decreases, the beam shrinks in the $x$ direction and expands in the $y$ direction, and as shown in row (b), as the quadrupole current decreases, particles escape from the beam core and appear to rotate in the halo region. The typical halo size is 2 or 3 times greater than that of the beam core.

\section{CONCLUSIONS AND OUTLOOK}

In this paper, we have presented a new high dynamic range method to image beam halo using a DMD. This technique can readily be applied to any accelerator or light source and provides a new, enabling technology for the study of halos and beam dynamics.

We have measured a DR $\sim 10^{5}$ of the imaging system using the UMER electron beam and a simple phosphor screen. This measured DR is not an inherent limit of the (a)

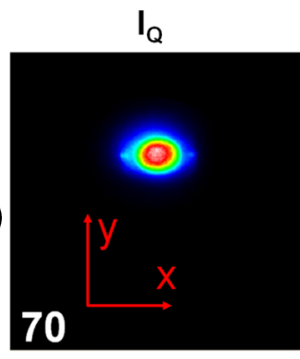

20000

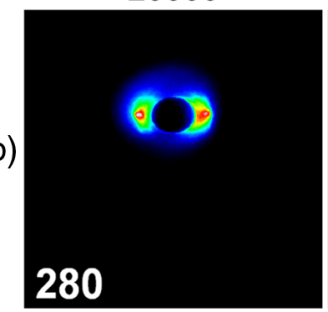

$82.9 \% \mathrm{I}_{Q}$

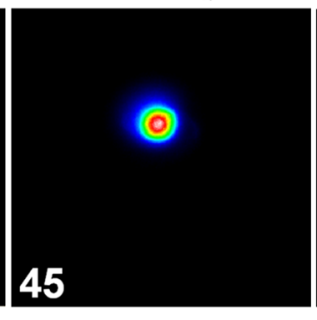

14000

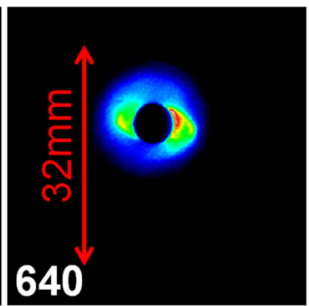

$66.3 \% \mathrm{I}_{\mathrm{Q}}$

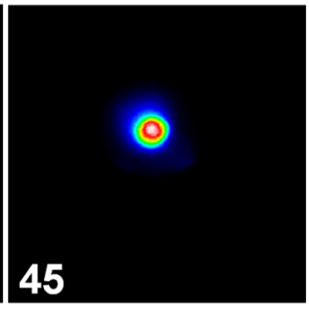

14000

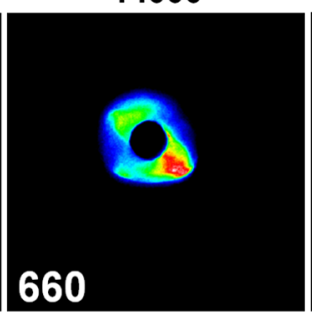

$49.7 \% l_{Q}$

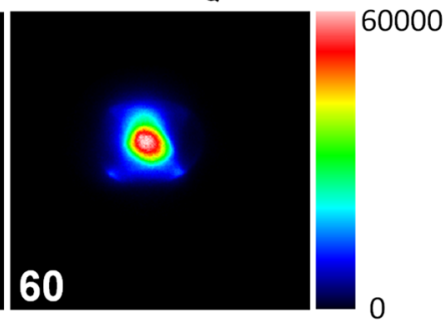

20000

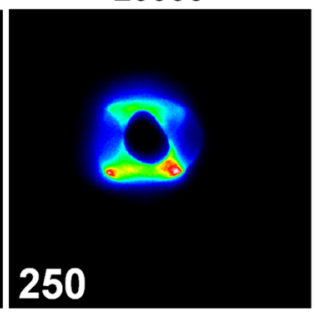

FIG. 18. Comparison of core and halo with changing quadrupole strength; (a) unmasked beam picture; (b) masked beam picture. 
method, but is primarily related to the maximum beam intensity achievable at UMER. In fact, preliminary results, using smaller, higher intensity beams, at both JLAB and SLAC/SPEAR3 have measured both higher beam $\left(>10^{5}\right)$ and system $\left(\sim 10^{7}\right)$ dynamic ranges.

We have shown that (1) the DMD has excellent extinction; (2) the quality of the beam image reflected from the DMD is unaffected by the diffraction effects produced by the DMD itself; and (3) the resolution of the DMD optics is equivalent to that of comparable system in which a mirror replaces the DMD.

We have also demonstrated the ability of DMD to create adaptive optical masks to block out the beam core, thus allowing us to observe the halo of the electron beam at UMER. We have shown the flexibility of this method in masking the beam core with different shapes and observed halo formation due to variation of the bias voltage in the electron gun and induced by varying the strength of an upstream quadrupole magnet.

Further halo studies at UMER and other accelerator facilities using upgrades to our current imaging system are already planned and will further improve the dynamic range and quality of the beam images obtained with this unique monitor. The results from these studies, when completed, will be presented in future papers.

\section{APPENDIX A: SCHEIMPFLUG COMPENSATION}

\section{Scheimpflug angle}

Scheimpflug compensation is used to correct the focus of an object tilted with respect to the lens plane. The effective lens optical layout for the second channel for our optical system is shown in Fig. 19, where line FDC is the light path of the central ray. Based on the Scheimpflug principle, which is commonly applied in photography [24], the planes of DMD, the effective lens, and the CCD sensor coincide at one point indicated as point $O$ in Fig. 19. The Scheimpflug angle can easily be determined from Fig. 19:

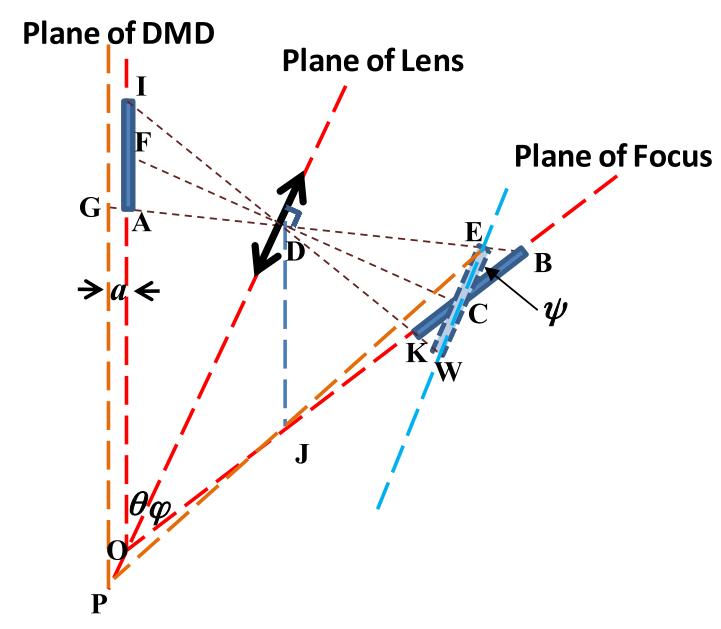

FIG. 19. Scheimpflug compensation diagram.

$$
\varphi=\arctan \left(\frac{v}{u} \tan \theta\right)
$$

where $u(v)$ is the distance between the effective lens and the object (image), indicated as line $F D(C D)$ in Fig. 19, and the image plane tilt angle for the DMD, $\theta=24^{\circ}$. For the second channel, $u+v=d=364 \mathrm{~mm}$, and $u / v=m_{c}=$ 1.033 , so that $u$ and $v$ are 185 and $179 \mathrm{~mm}$. Equation (A1) then gives $\varphi=24.7^{\circ}$.

\section{Depth of field}

An analysis of the Scheimpflug condition shows that if the source plane, in this case the plane of the DMD, is moved a distance $a$, the intersection point $O$ moves to point $P$ and the plane of focus is rotated around the point $J$, which is known as the "hinge point," such that the line EP lies in a new plane of focus. When Scheimpflug compensation is applied to view-camera photography, the camera system is designed so that the film plane can be physically rotated about the hinge point. However, in our experiments this is not convenient and, instead, we rotate the CCD sensor about its center in order to achieve the best focus for the second channel (see line ECW in Fig. 19). In doing so we empirically find that the best overall focus is achieved at the angle, $\varphi=24^{\circ}$, which is $0.7^{\circ}$ less than the value calculated $\left(24.7^{\circ}\right)$ from Eq. (A1).

Note that after rotation, the point $\mathrm{E}$ on the $\mathrm{CCD}$ will be out of focus the most. The plane EJP can then be used to estimate the maximum depth of field, $a$ shown in Fig. 19. The latter is related to the object distance $u$, the image distance $v$, the size of the DMD and the angle $\psi$, which is equal to the difference between the calculated Scheimpflug angle and the empirical angle. For our optical system, the size of DMD is $10.8 \mathrm{~mm}$ and $\psi=0.7^{\circ}$, so that $a=$ $0.06 \mathrm{~mm}$, i.e. the maximum depth of field is very small compared with the smallest dimension of the DMD.

\section{Distortion}

When Scheimpflug compensation is applied to the horizontal plane, there will be an unavoidable distortion due to the difference in magnifications in both the horizontal and vertical directions.

As shown in the top of Fig. 20, a square object with half size $s$ will transform to a trapezoid on the image plane. Because the vertical line $U V$ is perpendicular to the optical axis, the segment $U F$ and $F V$ will have the same magnification. However, for the horizontal line which is tilted by an angle $\theta=24^{\circ}$, as shown in the bottom of Fig. 20, the segments $I F$ and $A F$ will have different magnifications when they are imaged to lines $B C$ and $K C$, respectively. Moreover, the off-axis vertical segment $W I$ and $X A$ will have different magnifications when imaged to $W^{\prime} B$ and $X^{\prime} K$. We define the distortion

$$
D=\frac{K C}{B C}=\frac{X^{\prime} K}{W^{\prime} B} .
$$



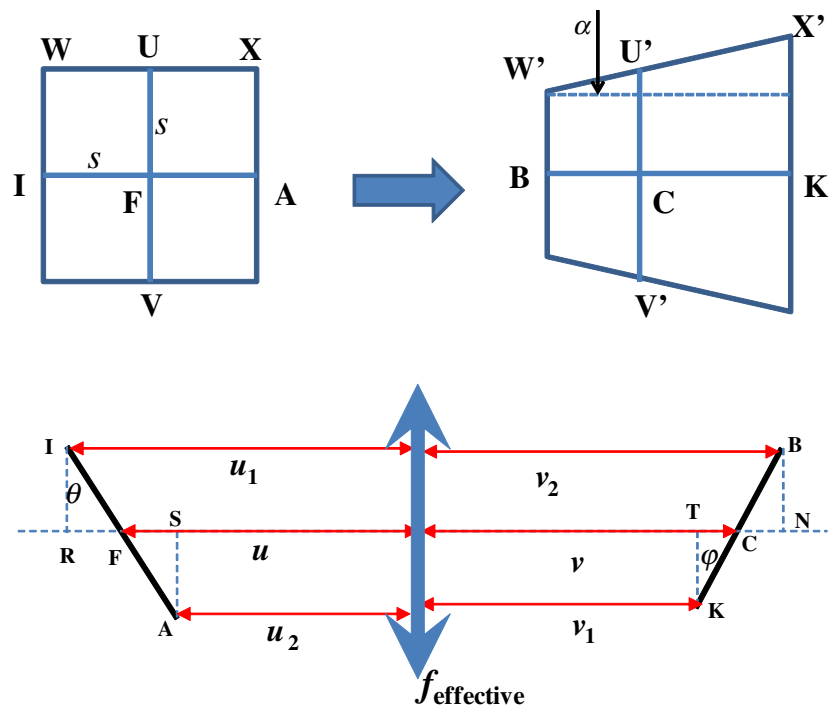

FIG. 20. Top: the effect of distortion due to Scheimpflug compensation. Bottom: diagram for calculation.

From Fig. 20,

$$
B C(K C)=\frac{B N(K T)}{\cos \varphi}=s m_{R C(S T)} \frac{\cos \theta}{\cos \varphi},
$$

where $m_{R C}$ and $m_{S T}$ are the magnifications of line segments $I R$ and $A S$, respectively, and

$$
m_{R C(S T)}=\frac{f_{\mathrm{eff}}}{u_{1(2)}-f_{\mathrm{eff}}}=\frac{f_{\mathrm{eff}}}{u \pm s \sin \theta-f_{\mathrm{eff}}},
$$

where $u_{1(2)}$, as shown in Fig. 20, are the source distances from the effective lens, and the subscripts 1, 2 refer to line segments $I R, A S$, respectively. Finally, we can express $D$ as

$$
D=\frac{m_{R C}}{m_{S T}}=\frac{u-s \sin \theta-f_{\text {eff }}}{u+s \sin \theta-f_{\text {eff }}}=\frac{\frac{f_{\text {eff }}}{m_{C} s}-\sin \theta}{\frac{f_{\text {eff }}}{m_{C} s}+\sin \theta},
$$

where $m_{c}$ is the magnification for the second channel. Equation (A5) shows that the distortion is significant only when the DMD size is magnified to a value comparable to the effective focal length, which is not the case for our system.

The trapezoid angle $\alpha$ can be determined from the geometry and

$$
\tan \alpha=\frac{s \sin \theta \cos \theta}{\sqrt{u^{2} \cos ^{2} \theta-2 u f_{\text {eff }} \cos \theta+f_{\text {eff }}^{2}}} .
$$

It can be easily verified that, when the tilted angle $\theta=0^{\circ}$, the distortion $D=1$ and trapezoid angle $\alpha=0$.

For the UMER optics, $u=179 \mathrm{~mm}, v=185 \mathrm{~mm}$, $s=5.4 \mathrm{~mm}$, and $f_{\text {eff }}=91 \mathrm{~mm}$, and $\theta=24^{\circ}$, so that the distortion rate $D=1.052$, and the trapezoid angle $\alpha=0.45^{\circ}$; therefore the distortion effect is negligible. This is verified by direct observation of a test target used in our bench tests, which consists of a gridded circle, whose diameter $(32 \mathrm{~mm})$ is the same as the phosphor screen used in our halo measurements.

In general, one always must compromise between the best focusing across the field of view and distortion when the DMD is used for imaging. A great aid to achieve this balance is the use of the checkerboard patterns mentioned above in the main text which can be written onto the DMD. Both the size and number of checkers is controllable by software. One can then use the edges and measured sizes of the checkers to simultaneously determine the focus as well as the distortion across the field of view.

\section{ACKNOWLEDGMENTS}

This work was supported by the Office of Naval Research and the DOD Joint Technology Office.

[1] D. Jeon, J. Stovall, A. Aleksandrov, J. Wei, J. Staples, R. Keller, L. Young, H. Takeda, and S. Nath, Phys. Rev. ST Accel. Beams 5, 094201 (2002).

[2] Y. Shimosaki and K. Takayama, Phys. Rev. E 68, 036503 (2003).

[3] C. K. Allen, K. C. D. Chan, P. L. Colestock, K. R. Crandall, R. W. Garnett, J. D. Gilpatrick, W. Lysenko, J. Qiang, J. D. Schneider, M. E. Schulze, R. L. Sheffield, H. V. Smith, and T. P.Wangler, Phys. Rev. Lett. 89, 214802 (2002).

[4] A. V. Fedotov, D. T. Abell, J. Beebe-Wang, Y. Y. Lee, N. Malitsky, J. Wei, and L. Gluckstern, in Proceedings of the European Particle Accelerator Conference, Vienna, 2000 (EPS, Geneva, 2000), p. 1289.

[5] R. H. Cohen, A. Friedman, S. M. Lund, A. W. Molvik, E. P. Lee, T. Azevedo, J.-L.Vay, P. Stoltz, and S.Veitzer, Phys. Rev. ST Accel. Beams 7, 124201 (2004).

[6] R. H. Cohen, A. Friedman, S. M. Lund, A. W. Molvik, T. Azevedo, J.-L. Vay, P. Stoltz, and S. Veitzer, Nucl. Instrum. Methods Phys. Res., Sect. A 544, 210 (2005).

[7] C.F. Papadopoulos, Ph.D. thesis, University of Maryland, College Park, 2009.

[8] M. Reiser, Theory and Design of Charged Particle Beams (Wiley, New York, 1994).

[9] R. L. Gluckstern, Phys. Rev. Lett. 73, 1247 (1994).

[10] T.P. Wangler, K. R. Crandall, R. Ryne, and T. S. Wang, Phys. Rev. ST Accel. Beams 1, 084201 (1998).

[11] R. A. Kishek, S. Bernal, I. Haber, H. Li, P. G. O'Shea, B. Quinn, M. Reiser, and M. Walter, AIP Conf. Proc. 693, 89 (2003).

[12] T. Shea and P. Cameron, AIP Conf. Proc. 693, 9 (2003).

[13] J. D. Gilpatrick, AIP Conf. Proc. 693, 122 (2003).

[14] P. Cameron, R. Connolly, R. Michnoff, V. Radeka, W. Ryan, T. Shea, R. Sikora, D. Stephani, S. Tepikian, and N. Tsoupas, in Proceedings of the 18th Particle Accelerator Conference, New York, 1999 (IEEE, New York, 1999), p. 2114.

[15] G. Haouat, N. Pichoff, C. Couillaud, J. Di Crescenzo, S. Joly, S. Sequin, and S. Striby, AIP Conf. Proc. 333, 245 (1995).

[16] C. P. Welsch, E. Bravin, B. Burel, T. Lefèvre, T. Chapman, and M. J. Pilon, Meas. Sci. Technol. 17, 2035 (2006); 
C. P. Welsch, E. Bravin, and T. Lefèvre, Proc. SPIE Int. Soc. Opt. Eng. 6616, 66160M (2007).

[17] T. Mitsuhashi, in Proceedings of the 9th European Particle Accelerator Conference, Lucerne, 2004 (EPS-AG, Lucerne, Switzerland, 2004), p. 2655.

[18] DMD Discovery 1100 Controller Board and Starter Kit, Texas Instruments Inc., 2005.

[19] J. Egberts and C.P. Welsch, JINST 5, P04010 (2010).

[20] H. D. Zhang, R. B. Fiorito, A. G. Shkvarunets, S. Bernal, I. Haber, R. A. Kishek, P. G. O'Shea, S. Artikova, and C. Welsch, in Proceedings of the 14th Beam Instrumentation Workshop, Santa Fe, New Mexico (LANL, Santa Fe, 2010), p. 543.

[21] R. A. Kishek, G. Bai, B. Beaudoin, S. Bernal, D. Feldman, R. Feldman, R. Fiorito, T.F. Godlove, I. Haber, T. Langford, P. G. O'Shea, B. Quinn, C. Papadopoulos, M.
Reiser, D. Stratakis, D. Sutter, K. Tian, J. C. T. Thangaraj, M. Walter, and C. Wu, in Proceedings of the 2007 Particle Accelerator Conference, Albuquerque, New Mexico (IEEE, New York, 2007), p. 820.

[22] R. B. Fiorito, A. Shkvarunets, H. D. Zhang, D. Douglas, F. G. Wilson, S. Zhang, J. Corbett, A. Fisher, W. Mok, K. Tian, and T. Mitsuhashi, in Proceedings of BIW12, Newport News, VA (2012), TUPG031.

[23] H. Zhang, R. Fiorito, A. Shkvarunets, J. Corbett, A. Fisher, and K. Tian, in Proceedings of IPAC12, New Orleans, LA (2012), WEOAA01.

[24] T. Scheimpflug, GB Patent No. 1196. Issued 1904; see also http://www.trenholm.org/hmmerk/\#SR.

[25] I. Haber, S. Bernal, R. A. Kishek, P. G. O'Shea, B. Quinn, M. Reiser, Y. Zou, A. Friedman, D. P. Grote, J.-L. Vay, Nucl. Instrum. Methods Phys. Res., Sect. A 519, 396 (2004). 